Canadian University Music Review

Revue de musique des universités canadiennes

\title{
La méthode Suzuki et l'approche de la langue maternelle
}

\section{Gilles Comeau}

Volume 22, numéro 2, 2002

URI : https://id.erudit.org/iderudit/1014509ar

DOI : https://doi.org/10.7202/1014509ar

Aller au sommaire du numéro

Éditeur(s)

Canadian University Music Society / Société de musique des universités canadiennes

ISSN

0710-0353 (imprimé)

2291-2436 (numérique)

Découvrir la revue

Citer cet article

Comeau, G. (2002). La méthode Suzuki et l'approche de la langue maternelle. Canadian University Music Review / Revue de musique des universités

canadiennes, 22(2), 113-126. https://doi.org/10.7202/1014509ar
Résumé de l'article

Dans la méthode Suzuki, un parallèle est proposé entre l'apprentissage de la langue maternelle et celui d'un instrument de musique. De ce rapprochement se dégage toute une série de principes qui orientent chacun des aspects de la méthode. Si le concept de l'apprentissage de la langue maternelle est une métaphore intéressante pour expliquer, en termes simples et imagés, la pédagogie privilégiée, un parallèle direct entre les deux types d'apprentissage suggère une simplicité illusoire de l'apprentissage de l'instrument. Cet article veut baliser les limites de la métaphore.
All Rights Reserved (C Canadian University Music Society / Société de musique des universités canadiennes, 2003
Ce document est protégé par la loi sur le droit d'auteur. L'utilisation des services d'Érudit (y compris la reproduction) est assujettie à sa politique d'utilisation que vous pouvez consulter en ligne.

https://apropos.erudit.org/fr/usagers/politique-dutilisation/ 


\section{LA MÉTHODE SUZUKI ET L'APPROCHE DE LA LANGUE MATERNELLE}

\section{Gilles Comeau}

La méthode Suzuki est l'une des approches dominantes dans l'enseignement instrumental. Elle s'est imposée très rapidement partout en Amérique du Nord. Alors que de nombreux livres et articles ont été écrits dans le but de présenter la méthode et d'en faire connaître les mérites ${ }^{1}$, il n'existe, à notre connaissance, aucune étude critique portant sur les principes orientant cette approche ${ }^{2}$. Cette absence de recherche critique peut s'expliquer en partie par le fait que les spécialistes de l'approche Suzuki sont avant tout des convertis à la méthode et s'emploient davantage à en faire la promotion qu'à mettre en question certaines de ses orientations. De plus, le contrôle rigoureux exercé par la Suzuki Association of the Americas et par l'éditeur des cahiers de la méthode, Warner Bros., a empêché, à quelques occasions, la publication de certains ouvrages ou exigé la modification du contenu de certains autres. Pourtant, la recherche ne peut se soustraire à l'obligation de mieux comprendre les principes éducatifs en vigueur, d'en souligner l'intérêt, mais aussi les limites. Cette étude a pour objet d'examiner le principe qui est au cœur même de la méthode Suzuki, à savoir : l'apprentissage d'un instrument de musique peut-il s'effectuer sur le modèle de la langue maternelle?

Ce principe, appelé mother-tongue approach (approche de la langue maternelle) veut que l'apprentissage initial se fasse par l'oreille. Cette proposition

1 Shinichi Suzuki est l'auteur de : Ability Development From Age Zero (Athens : Senzay, 1969); How to Teach Suzuki Piano (Secausus, New Jersey : Summy-Birchard Music, 1993); Nurtured by Love. A New Approach to Education (New York : Exposition Press, 1969); Talent Education for Young Children (1969, version japonaise; New Albany : World-Wide Press, 1986). Les contributions d'autres auteurs comprennent : Carole L. Bigler et Valery Lloyd-Watts, Studying Suzuki Piano: More than Music (Athens : Senzay, 1979); Gilles Comeau (dir.), Teaching Suzuki Piano. 10 Master Teachers' Viewpoints (Ottawa : CFORP, 1997); Evelyn Hermann, Shinichi Suzuki: The Man and His Philosophy (Secausus, New Jersey : Summy-Birchard Music, 1981); Haruko Kataoka, Thoughts on the Suzuki Piano School (Princeton : Birch Tree Group Ltd, 1985); John D. Kendall, The Suzuki Violin Method in American Music Education (Secausus, New Jersey : Warner Bros., 1978); Doris Koppelman, Introducing Suzuki Piano (San Diego : Dichter Press, 1978); Elizabeth Mills et $S^{r}$ Therese Cecile Murphy, The Suzuki Concept : An Introduction to a Successful Method for Early Music Education (San Francisco : Diablo Press, 1973); Mary Craig Powell, Focus on Suzuki Piano (Secausus, New Jersey : Warner Bros., 1988); Constance Starr et William Starr, To Learn With Love (Secausus, New Jersey : Warner Bros., 1983).

$2 \AA$ titre d'exemple, Dissertation Abstracts International présente plusieurs thèses portant sur la méthode Suzuki : certaines font connaître les bases de la méthode ainsi que les influences qui ont orienté l'élaboration de la méthode; d'autres cherchent des éléments quantitatifs pour appuyer les bienfaits de la méthode; et d'autres s'intéressent à l'adaptation de la méthode à de nouveaux instruments ou à de nouveaux contextes. Mais, parmi toutes ces thèses, aucune ne propose une analyse critique des principes sous-jacents à la méthode Suzuki. 
n'est pas nouvelle. Déjà au XVIII' siècle, dans son traité pédagogique sur L'art de toucher le clavecin, Couperin reconnaît l'importance de s'initier au jeu du clavier par «l'oreille» :

On devroit ne commencer à montrer la tablature aux enfants qu'après qu'ils ont une certaine quantité de pièces dans les mains. Il est presqu'impossible, qu'en regardant leur Livre, les doigts ne se dérangent; et ne se contorsionnent : que Les agrémens même n'en soient altèrés; d'ailleurs, La memoire se forme beaucoup mieux en aprenant par-cœur ${ }^{3}$.

Ce principe est resté en vigueur au cours des siècles et on le retrouve au $\mathrm{XX}^{\mathrm{e}}$ chez de grands pédagogues nord-américains. Pour Abby Whiteside, « jouer à l'oreille est la seule bonne façon d'apprendre pour un élève débutant. Pour s'en convaincre, il n'est qu'à observer l'aisance, la précision et la justesse des élèves qui commencent de cette manière ${ }^{4}$ ». Whiteside répétera souvent que « l'élève en piano doit commencer en jouant à l'oreille ${ }^{5}$ » et que l'apprentissage de la lecture doit venir en second. Pour Marc Durand, il est important de ne pas présenter la lecture au début des études musicales, car on surcharge l'enfant ${ }^{6}$. L'apprentissage du piano doit commencer par le son; il faut établir un lien entre l'oreille et le clavier avant d'établir une connexion entre l'œil et le clavier. La lecture musicale doit s'enseigner séparément, comme une habileté en soi.

L'apprentissage par oreille est aussi au cœur de la méthode Suzuki, mais " l'approche de la langue maternelle » développée par Shinichi Suzuki va beaucoup plus loin, puisque celui-ci dégage, à partir de ce principe, une série d'orientations qui affectent tous les différents aspects de sa méthode. Or, la recherche n'a encore jamais démontré si un rapprochement direct entre ces deux types d'apprentissage est véritablement fondé ou si, au contraire, il peut conduire à des conceptions erronées et à des pratiques illusoires. Mon hypothèse est la suivante : le concept de l'apprentissage de la langue maternelle est une métaphore intéressante pour expliquer en termes simples et imagés une orientation pédagogique privilégiée, mais un parallèle direct entre les deux types d'apprentissage est beaucoup trop simpliste. J'exposerai d'abord le principe formulé par Suzuki et par les spécialistes de la méthode, afin d'en comprendre toutes les ramifications. Ensuite, j'examinerai les théories présentement en vigueur pour expliquer le processus d'apprentissage de la langue maternelle. Je mentionnerai ensuite comment la pédagogie de la lecture musicale a tenté de se modeler sur les principes de l'apprentissage de la langue

3François Couperin, L'Art de toucher le clavecin (1717; reproduction, Van Nuys, Calif. : Alfred Publishing Co. 1974), 32.

4 \& The only safe beginning for a music student is to play by ear. To believe this completely, one needs only observe the ease and accuracy of those students who began in that manner $m$. Cette traduction et toutes les suivantes sont de l'auteur de l'article. Abby Whiteside, Mastering the Chopin Etudes and Other Essays (1969; reproduction avec d'autres essais sous le titre On Piano Playing, Portland, Oreg. : Amadeus Press, 1997), 165.

5 « The music student should begin by playing by ear $\%$. Ibid., 157.

6Marc Durand, « Approche psycho-pédagogique de l'enseignement du piano » (Montréal : Université de Montréal, 1996). Cours de pédagogie du piano donné a l'Université de Montréal. 
maternelle. Je dégagerai enfin ce qui peut soit appuyer, soit remettre en question le premier principe de la méthode Suzuki.

\section{LE PRINCIPE DE LA MÉTHOdE SUZUKI ET SES COROLLAIRES}

Au milieu du $\mathrm{XX}^{\mathrm{e}}$ siècle, Shinichi Suzuki, alors professeur de violon au Japon, est émerveillé par le fait que tous les enfants parviennent à apprendre leur langue maternelle avec précision et exactitude, avec toutes les nuances d'inflexion qui caractérisent les différents dialectes japonais ${ }^{7}$. Il remarque également que l'acquisition de cette habileté complexe qu'est la maîtrise d'une langue s'effectue très naturellement ${ }^{8}$. Il conclut alors que l'approche par laquelle les enfants apprennent à parler est la méthode la plus efficace pour apprendre la musique : "On élève les enfants du monde entier à l'aide d'une approche pédagogique parfaite: leur langue maternelle. Pourquoi ne pas appliquer cette méthode à d'autres habiletés? J'ai eu le sentiment que je venais de faire une découverte formidable ${ }^{9} »$. Pour Suzuki, la méthode d'enseignement de la langue maternelle peut parfaitement s'appliquer à d'autres secteurs : «Il n'y a pas de différence entre les types de potentiel humain, que ce soit le langage ou autre chose. Les possibilités deviennent des habiletés, là où il y a stimulation et entraînement ${ }^{10}$ ».

Si tous les enfants parviennent à apprendre une langue, Suzuki en conclut que tous ont, au départ, d'énormes capacités et que les principales causes qui limitent le potentiel des enfants sont un enseignement de mauvaise qualité, un environnement appauvri, ou des attentes inadéquates de la part des adultes ${ }^{11}$. Il faut donc remettre en question la conception selon laquelle les résultats de l'éducation reposent sur des habiletés innées : «Une oreille musicale n'est pas innée. C'est une aptitude humaine qui se développe à partir de l'écoute ${ }^{12} »$. Après avoir observé comment l'enfant apprend sa langue maternelle, Suzuki formule les conclusions suivantes :

1. Les humains ne naissent pas avec un talent particulier, mais avec le potentiel de le développer. 2. [...] La spécificité de ce potentiel est de croître grâce à la stimulation et à l'entraînement répété. [...] 3. Quel que soit le champ d'activités, un entraînement approprié bien supervisé et un environnement adéquat permettront de former des habiletés exceptionnelles ${ }^{13}$.

7 Suzuki, Ability Development From Age Zero, 4.

8 Suzuki, Talent Education for Young Children, 11.

9 « All children everywhere in the world are brought up by a perfect educational method: their mother tongue. Why not apply this method to other faculties? I felt I made a tremendous discovery ». Suzuki, cité par Hermann, Shinichi Suzuki: The Man and His Philosophy, 19.

10 \% There is no distinction between one kind of human potential and another: it is the same whether it is language or anything else. Potentials grow as abilities where stimulation and training lead ». Suzuki, Ability Development From Age Zero, 11.

11 Suzuki, Ability Development From Age Zero, 1.

12 « An ear for music is not innate. It is a human aptitude which can only be developed by listening ". Mills et Murphy, The Suzuki Concept, 12.

13 « 1 . Human beings are not born with particular talents, but have the potential in which those talents originate. 2. [Its] property is to grow by stimulation and by repeated training through stimulation. [...] 3. No matter what area we are talking about, if proper training is given under good leadership and 
Cela ne veut pas dire que tous les enfants soient nés avec des capacités identiques. De toute évidence le potentiel humain varie d'une personne à l'autre, et cette variation va influencer le degré d'intensité avec lequel un individu pourra répondre aux stimuli de son environnement. Toutefois, même en présence d'un potentiel moindre, n'importe quelle habileté pourra atteindre un haut niveau de croissance, comme cela se produit avec l'acquisition de la langue maternelle ${ }^{14}$. C'est pourquoi Suzuki a appelé sa méthode "Éducation du talent ». De plus, "l'idée de langue maternelle implique que c'est l'environnement et non l'hérédité qui éduque l'enfant ${ }^{15}$ », et « le développement des habiletés est directement proportionnel à la qualité de l'environnement ${ }^{16} »$. La méthode Suzuki est donc soucieuse de créer un environnement optimal qui favorise le développement musical de l'enfant, en prenant exemple sur le processus d'acquisition du langage. À sa naissance, le bébé baigne dans un environnement où il entend constamment parler. Il absorbe et intériorise sa langue première selon un processus naturel. Suzuki conclut alors que « si l'enfant était constamment exposé à la musique, autant qu'il l'a été depuis sa naissance au langage, il développerait des habiletés musicales tout aussi remarquables ${ }^{17}$ ». L'écoute de musique constitue donc un facteur déterminant : avant d'apprendre à jouer d'un instrument, les enfants doivent absorber la musique par l'écoute répétée des pièces qu'ils vont prochainement jouer. Plus l'enfant " aura intériorisé la musique, plus il lui sera facile de la reproduire à l'instrument ${ }^{18}$ ".

Au début du programme Suzuki, l'apprentissage se fait donc à l'oreille et respecte toujours le principe selon lequel l'enfant apprend d'abord à parler avant de s'initier à la lecture et à l'écriture : « D'abord parler - ensuite lire. D'abord jouer - ensuite lire ${ }^{19}$ ». Le processus d'acquisition de la langue maternelle est l'élément justificateur d'un apprentissage par oreille.

Personne n'apprend à un bébé à parler en l'initiant aux caractères d'imprimerie et aux mots écrits. L'ordre naturel est d'enseigner l'alphabet et la lecture après qu'un enfant a appris à parler. Ainsi, nous n'utilisons pas de musique imprimée lorsque nous enseignons le piano à des enfants d'âge préscolaire; nous leur demandons plutôt d'apprendre de nouvelles chansons à partir de l'écoute d'enregistrements et nous leur apprenons à positionner leurs doigts ${ }^{20}$.

in a good environment which makes it easy to grow, any talent will display outstanding ability ». Suzuki, Talent Education for Young Children, 11.

14 Ibid., 12.

15 « The idea of mother tongue [...] implies that environment and not heredity educates children ". Bigler et Lloyd-Watts, Studying Suzuki Piano, 2. Ce sont Bigler et Lloyd-Watts qui soulignent.

16 " The development of those abilities is directly dependent on the quality of his environment ". Doris Harrel, « Philosophy », dans Teaching Suzuki Piano, dir. par Comeau, 7.

17 \& Children are surrounded by these language sounds from birth, and Suzuki reasoned that if the children were surrounded by musical sounds to the same degree, they would develop an equally remarkable ability in music ». Bigler et Lloyd-Watts, Studying Suzuki Piano, 1.

18 « [...] the more thoroughly it is internalized, the easier it is to reproduce ". Kataoka. Thoughts On the Suzuki Piano School, 13.

19 « Speak first-read later. Play first-read later $\%$. C. Starr et W. Starr, To Learn with Love, 188.

20 « Nobody teaches a baby to talk by starting with printed letters and words. The natural order is to teach letters and reading after a child learns to talk. In the same manner, in teaching piano to preschool 
L'apprentissage de la lecture est important, mais il sera introduit ultérieurement : «Attendez l'âge et le moment approprié pour enseigner [la lecture] aux enfants. Avant cette période, il est plus important de développer l'oreille des enfants afin qu'ils puissent écouter et évaluer les sons qu'ils produisent $[\ldots]^{21} »$. $\mathrm{Au}$ début, l'enfant doit véritablement écouter s'il veut être capable de jouer puisqu'il n'a pas de partitions pour le guider. Pour chaque nouvelle pièce, il doit se rappeler les mélodies et les harmonies entendues ${ }^{22}$. Les progrès de l'enfant dépendent alors directement du temps passé à l'écoute d'enregistrements ${ }^{23}$.

En s'inspirant du modèle d'apprentissage de la langue maternelle, la méthode Suzuki prône une initiation précoce à la musique, car l'oreille aura de meilleures chances de se développer si son éducation commence tôt : "C'est entre la naissance et l'âge de 7 ans que se situe la meilleure période pour développer l'oreille de l'enfant ${ }^{24} »$. De plus, selon le principe de l'apprentissage de la langue maternelle, il ne devrait y avoir aucun échec. N'importe quel enfant capable d'apprendre sa langue maternelle a nécessairement la capacité d'apprendre à jouer d'un instrument. Dans l'apprentissage d'une langue maternelle, tous atteignent un haut niveau de réussite. Personne n'a l'impression que c'est difficile, personne n'abandonne à mi-chemin, personne n'échoue : il devrait en être ainsi de l'apprentissage instrumental ${ }^{25}$. La conviction que tous les enfants ont la capacité de jouer d'un instrument de musique selon une approche positive et naturelle est l'une des contributions majeures de la méthode Suzuki au domaine de l'apprentissage instrumental.

\section{LES THÉORIES PSYCHOLOGIQUES DE L'APPRENTISSAGE DE LA LANGUE MATERNELLE}

Depuis longtemps, on explique l'acquisition de la langue maternelle par un processus d'imitation et de renforcement. L'enfant apprend à parler en « copiant » ce qu'il entend autour de lui et ses réponses sont renforcées par les réactions des adultes. Mais au cours des dernières décennies, il est devenu évident que le principe d'imitation ne parvient pas à expliquer à lui seul le développement de la langue maternelle. Certes, l'enfant imite beaucoup et il apprend ainsi les phonèmes et le vocabulaire de sa langue, mais les habiletés grammaticales propres à l'acquisition d'une langue ne peuvent s'expliquer par un simple processus d'imitation. Depuis la fin des années 1950, des théories concernant une prédisposition pour l'apprentissage de la langue ont commencé à s'imposer. Ainsi, le nouveau-né posséderait les structures d'une grammaire générative, ce que Chomsky nomme la " grammaire universelle ${ }^{26}$ ». Le cer-

children, we do not use printed music, but rather have them learn new songs from listening to the record and showing them how to use their fingers ». Suzuki, How To Teach Suzuki Piano, 11.

21 « Wait to teach the student until an appropriate age and time. Until that time, I think it's more important to develop the ear so that children listen to and judge their own sound [...] ». Ibid., 12.

22Koppelman, Introducing Suzuki Piano, 45.

23C. Starr et W. Starr, To Learn With Love, 125.

24 « It is believed that the ability of a child to learn aurally is strongest from birth to age seven ". Mary Craig Powell, « Philosophy », dans Teaching Suzuki Piano, dir. par Comeau, 6. 
veau humain aurait donc une prédisposition au langage et dès que l'enfant entend parler autour de lui, certains principes génératifs se mettent automatiquement en opération. C'est ainsi que l'enfant parvient à décoder différents phonèmes et à élaborer des hypothèses sur la construction de la phrase. Cette connaissance lui permet ensuite, en procédant par essais et erreurs, de formuler ses propres phrases et d'être compris par d'autres. Quoique les opinions different quant aux spécificités de cette grammaire générative, bon nombre de psychologues admettent que seul un principe inné du langage permet d'expliquer : 1) la vitesse phénoménale à laquelle les enfants apprennent à parler; 2) la possibilité pour l'enfant de créer de nouvelles phrases; 3) l'indépendance relative entre les habiletés langagières et l'intelligence; et 4) la similarité dans l'acquisition des structures grammaticales chez les enfants de différents milieux et différentes cultures. L'approche cognitive de l'acquisition du langage, quant à elle, met plutôt l'accent sur le développement intellectuel de l'enfant. Ainsi, les structures linguistiques ne peuvent faire surface que si certains développement cognitifs sont déjà en place. Les capacités initiales pour l'acquisition d'une langue, présentes chez tout être humain, seraient organisées en stades successifs. Plusieurs études ont tenté d'établir des liens entre les stades de développement cognitifs, entre autres les stades établis par Piaget, et l'émergence des habiletés linguistiques ${ }^{27}$.

À toutes ces théories basées sur l'imitation, la grammaire générative ou le développement cognitif, il faut ajouter un facteur longtemps minimisé, soit l'importance de l'environnement. Même si l'environnement linguistique n'a pas à être très riche et qu' aucun enseignement n'est requis pour que l'acquisition du langage s'effectue, il est néanmoins nécessaire que le bébé soit en contact direct avec un adulte parlant. Il est communément admis, même si cela n'est appuyé par aucune expérience scientifique, que le son de la radio ou de la télévision n'est pas suffisant pour permettre à l'enfant d'apprendre à parler. Une interaction avec les adultes qui l'entourent s'impose pour éveiller et maintenir chez le bébé le désir de parler. La même interaction est requise pour la communication gestuelle du langage signé chez les enfants sourds. De plus, les parents ont tendance à adopter un type de langage particulier lorsqu'ils s'adressent à leur bébé : simplification des contenus et des constructions grammaticales (phrases très courtes), débit de voix plus lent, registre de voix plus aigu, utilisation de mots spéciaux ou d'onomatopées qui accentuent le lien affectif de la communication. Tous ces facteurs auraient un impact sur l'apprentissage de la langue chez l'enfant. L'environnement social serait donc un élément capital du processus d'acquisition de la langue maternelle. Dans l'état actuel de la recherche, on doit tenir compte de bien des facteurs pour expliquer l'acquisition de la langue et il s'avère impossible de choisir une seule théorie. Il serait plus juste de considérer que l'imitation, certaines structures gramma-

27 Voir Bénédicte de Boysson-Bardies, Comment la parole vient aux enfants (Paris : Odile Jacob, 1996); Paul Bloom. Language Acquisition (1993; Cambridge : The MIT Press, 2000). 
ticales innées, le développement cognitif et l'environnement social jouent tous un rôle dans le processus d'acquisition de la langue première.

\section{APPRENTISSAGE DE LA LECTURE SELON LE PRINCIPE DE L'APPRENTISSAGE DE LA LANGUE MATERNELLE}

À l'époque où Suzuki développait une méthode d'enseignement de la musique qui s'inspirait de l'acquisition de la langue maternelle, des éducateurs s'intéressaient au même principe pour l'enseignement de la lecture. Fascinés par la facilité avec laquelle tous les enfants normaux parviennent à un très bas âge à maîtriser une activité aussi complexe qu'apprendre à parler, et remarquant que cette habileté s'acquiert selon un processus naturel, des spécialistes de l'enseignement de la lecture se sont demandé si les caractéristiques reliées à l'apprentissage de la langue pourraient s'appliquer à celui de la lecture en salle de classe. Don Holdaway s'est intéressé à ce qu'il a appelé le developmental learning, en référence à ce type d'apprentissage pratiqué par le jeune enfant avant son arrivé à l'école ${ }^{28}$. Cet apprentissage ne requiert qu'un minimum d'instruction et ne fait appel à aucun professionnel de l'enseignement. Selon Holdaway, l'entraînement s'opère avec une telle facilité et nécessite si peu d'enseignement planifié qu'Holdaway est porté à qualifier ce type d'apprentissage de «naturel » pour le distinguer de celui des habiletés qui nécessitent ou semble nécessiter - un enseignement intensif. Tous les enfants parviennent à maîtriser certaines habiletés, comme apprendre à parler, en étant simplement exposés à un environnement approprié. Les parents deviennent des modèles que le jeune enfant tente d'imiter. Les premiers efforts de l'enfant ne sont qu'une approximation de l'habileté désirée; mais, grâce aux répétitions, l'enfant affine sa performance et s'approche de plus en plus de l'objectif recherché. D'après Holdaway, le developmental learning aurait les caractéristiques suivantes : 1) immersion dans un environnement où l'habileté à acquérir est sollicitée adéquatement, où l'on favorise l'imitation au lieu de l'instruction et où l'on fournit de nombreux exemples de l'habileté à développer; 2) renforcement immédiatement après chaque essai, même si le résultat est encore très approximatif; 3) approche essentiellement autorégulatrice : l'implication dans la tâche, la durée des essais et le rythme de progression sont contrôlés en bonne partie par l'enfant qui pratique l'habileté à développer, que l'adulte soit présent ou non; et 4) environnement sécuritaire, positif et non compétitif, qui n'offre aucune menace à l'égard de la tâche à maîtriser et qui respecte les écarts parfois considérables entre les différents enfants. Holdaway est convaincu que la lecture et l'écriture peuvent s'apprendre en appliquant ces conditions. En s'appuyant sur le fait que tous les enfants veulent apprendre à lire et en se basant sur son expérience de clinicien auprès de jeunes enfants qui éprouvaient de la difficulté à lire et à écrire, il pense que tous les jeunes élèves ont la capacité d'apprendre par eux-mêmes, selon un processus d'apprentissage naturel, s'ils bénéficient d'un environnement approprié. 
Brian Cambourne s'est intéressé au même phénomène, qu'il a appelé le natural learning ${ }^{29}$. Il explique que si l'apprentissage de la lecture ne s'opère habituellement pas de façon aussi naturelle que l'apprentissage de la langue, cela est dû au type d'environnement dans lequel baigne le jeune enfant. L'environnement est riche et adéquat pour l'acquisition de la langue, mais offre rarement les mêmes conditions pour l'apprentissage de la lecture et de l'écriture. Pour Cambourne, l'école a la responsabilité de créer cet environnement en mettant en place les conditions qui vont permettre à ces habiletés de se développer en suivant l'approche utilisée pour l'acquisition de la langue parlée. Cambourne a identifié les composantes d'une « approche naturelle » appliquée à la lecture et à l'écriture : 1) choix de textes appropriés; 2) démonstrations multiples de la façon dont les textes sont construits et utilisés; 3 ) responsabilisation de l'enfant pour décider ce qu'il va lire et écrire, quand, comment il le fera; 4) création d'attentes chez l'enfant dans le but de l'encourager à compléter les tâches entreprises; 5) allocation du temps nécessaire pour s'investir dans des activités de lecture et d'écriture; 6) occasion d'employer la lecture et l'écriture dans des contextes significatifs; 7) liberté de produire approximativement l'habileté requise, étant donné que les erreurs font partie du processus d'apprentissage; et 8 ) soutien de l'enfant et information concernant ses progrès de la part des adultes maîtrisant ces habiletés ${ }^{30}$.

\section{RAPPROCHEMENTS ENTRE L'APPRENTISSAGE DE LA LANGUE MATERNELLE ET L'APPRENTISSAGE DE LA MUSIQUE}

Il est possible de faire plusieurs rapprochements entre l'apprentissage de la langue maternelle et l'apprentissage de la musique. L'acquisition de la langue s'opère selon un processus naturel : la langue ne s'enseigne pas, l'être humain naît avec une aptitude à parler. L'acquisition de la culture musicale première s'opère aussi selon un processus naturel. On a beaucoup étudié le développement de l'expression vocale et des réactions corporelles en présence de la musique chez le jeune enfant. Ces formes d'expression ne nécessitent aucun enseignement, mais sont le résultat d'un processus d'acquisition, qui se développe naturellement au contact d'autres membres dans un environnement culturel donné. Comme on l'a vu, les théories d'acquisition de la langue ont également proposé l'existence d'une prédisposition génétique à la parole, une grammaire universelle; la recherche en musique formule elle aussi une explication semblable :

Si beaucoup d'aspects de la musique ont une spécificité culturelle, les règles générales qui régissent son analyse, sa mise en mémoire et sa reproduction semblent déjà universelles. Actuellement [...] de fortes présomptions permettent de penser qu'il existe, dès la naissance, des circuits préprogrammés pour la musique, qui en feraient un besoin physiologique à part entière ${ }^{31}$.

29Brian Cambourne, The Whole Story: Natural Learning and the Acqusition of Literacy in the Classroom (Auckland : Ashton Scholastic, 1988).

30 Ibid., 33.

31 Claude-Henri Chouard, L'oreille musicienne. Les chemins de la musique de l'oreille au cerveau 
À la naissance, le bébé est en mesure d'apprendre n'importe quelle langue et seul son environnement social va déterminer pourquoi il développera la maîtrise de telle langue et non de telle autre. L'enfant va ainsi acquérir une seule langue maternelle (ou dans le cas de familles bilingues, deux langues premières). De la même façon, le bébé naissant possède les atouts nécessaires pour absorber n'importe quel langage musical, et seul son environnement immédiat déterminera quel univers sonore deviendra sa culture musicale première. À la suite du processus d'acculturation des premières années, toute autre culture musicale apparaîtra désormais " étrangère ».

Il est clair aussi qu'il y a des périodes critiques pour l'acquisition de certaines aptitudes linguistiques. Ces périodes sont déterminées par la perte d'une certaine plasticité lorsque les connecteurs des neurones deviennent spécialisés. La recherche actuelle permet de croire que la période critique pour l'acquisition de la langue maternelle se termine à 7 ans $^{32}$. Un enfant qui n'aurait pas entendu parler avant cet âge serait incapable d'apprendre à parler convenablement par la suite. La période critique pour l'apprentissage d'une langue étrangère se terminerait à l'adolescence : au-delà de cette période, la langue étrangère sera toujours parlée avec un accent. Depuis longtemps, la psychologie de la musique a démontré l'existence de périodes critiques pour l'acquisition de certaines habiletés. La petite enfance est une période très favorable au développement des habiletés auditives. Très vite les compétences du nourrisson deviennent impressionnantes. Par exemple, dès le huitième mois, l'enfant est capable de reconnaître la notion d'octave ${ }^{33}$. La recherche a également démontré que le développement de l'oreille absolue a besoin, en plus d'un don inné, d'une connaissance de la musique à un âge très précoce ${ }^{34}$. Ainsi, tout semble indiquer que le très jeune enfant est capable de profiter très tôt d'un environnement musical sophistiqué, même si pendant les premières années de sa vie il ne pourra reproduire que les rythmes et les mélodies les plus simples. La plasticité cérébrale rend compte, comme dans l'acquisition du langage, de tous les phénomènes d'apprentissage musical :

Dans ce domaine, [la plasticité cérébrale] contribue sans doute à la création de réseaux neuronaux, qui réalisent des structures capables de traiter des groupes de fréquences sonores correspondant à une note, ou un groupe de notes constituant un accord. Les mécanismes de la plasticité cérébrale expliquent également l'origine culturelle de ce qui apparaît comme « obligatoire » dans la plupart des systèmes musicaux. Ainsi s'explique le rôle fondamental d'un environnement musical précoce dans le développement des aptitudes et de la culture musicales, parce qu'il permet une programmation aisée de ces formations auditives ${ }^{35}$.

(Paris : Gallimard, 2001), 308.

32 Boysson-Bardies, Comment la parole vient aux enfants, 308.

33 Marsha G. Clarkson et Rachel K. Clifton, « Infant Pitch Perception: Evidence for Responding to Pitch Categories and the Missing Fundamental ", Journal of the Acoustical Society of America, 77, n 4 (avril 1985) : 1521-28.

34Chouard, L'oreille musicienne, 255.

35 Ibid., 214. 
Alors qu'on a pensé pendant longtemps que la musique était un champ culturel sans importance vitale pour l'humain, ou encore le sous-produit d'une fonction cérébrale plus importante, tel le langage, on reconnaît maintenant à la musique un caractère physiologique à part entière : "De nombreux scientifiques estiment aujourd'hui que l'écoute et l'expression musicales représentent une propriété physiologique qui n'appartient qu'à l'homme, au même titre que la parole, et qui lui est tout autant indispensable, même si son organisation cérébrale s'en différencie nettement ${ }^{36}$ ». Les fonctions biologiques de communication liées aux sens sont multiples, et la musique revendique de plus en plus une place à part entière : « En considérant l'audition, la spécialisation de certains centres cérébraux pour la parole est certes remarquable, mais il faut surtout s'étonner de notre penchant naturel pour écouter de la musique, pour en jouer et jouir de l'aspect affectif qui l'accompagne ${ }^{37}$ ».

\section{DIFFÉRENCES ENTRE APPRENDRE LA MUSIQUE ET APPRENDRE À JOUER D'UN INSTRUMENT}

Le jeune enfant a une prédisposition instinctive à développer la parole et, comme nous l'avons vu, cette tendance serait inscrite dans le code génétique de tout bébé. Il est possible de créer un parallèle avec l'apprentissage musical et de penser que tout jeune enfant a une prédisposition à la musique et qu'il va acquérir, selon un processus naturel, les caractéristiques musicales de base de sa culture. Toutefois, il faudrait bien distinguer entre " apprendre la musique » et s'initier à la musique savante par l'apprentissage du chant classique ou d'un instrument. S'initier à une culture musicale, apprendre à chanter le répertoire folklorique et populaire, développer un intérêt pour l'appréciation musicale sont toutes des compétences qui s'acquièrent naturellement par tous les enfants du monde, dans la mesure où la musique est présente dans leur environnement. Ces habiletés sont reliées à un apprentissage naturel et se manifestent de façon spontanée. Cet accomplissement est de nature bien différente des habiletés requises dans l'interprétation de la musique savante dont l'acquisition est possible grâce à un ensemble d'activités reliées à la pratique vocale ou instrumentale. Mais cette pratique requiert la maîtrise de nombreux procédés techniques au moyen d'une vaste quantité de répétitions, un souci constant de raffinement et une analyse minutieuse des résultats. De plus, il faut déployer un effort pédagogique considérable pour mener les enfants à un stade où la maîtrise technique et la lecture de la musique sont suffisamment assimilés.

Il est important de distinguer l'acculturation de la formation. La première s'élabore selon un processus d'acquisition naturelle, comme l'apprentissage de la langue maternelle, tandis que la seconde nécessite un processus d'apprentissage structuré et supervisé. Selon Chouard, les caractéristiques de l'acculturation seraient les suivantes : 
En premier lieu, un ensemble commun de capacités primitives, présentes dès la naissance ou tout de suite après celle-ci. En deuxième lieu, vient un ensemble commun d'expériences fournies par la culture au fur et à mesure que les enfants grandissent. En troisième lieu, il nous faut mentionner l'impact d'un système cognitif général qui se modifie rapidement au fur et à mesure de l'acquisition des nombreuses autres compétences appuyées par la culture. Ces éléments se combinent pour produire une séquence d'accomplissements qui est, grosso modo, identique pour la majorité des enfants d'une culture, et un ensemble d'âges, grosso modo identiques, auxquels les divers accomplissements interviennent ${ }^{38}$.

Ainsi, l'acculturation s'opère sans un effort conscient de la part de l'enfant. Par exemple, les jeunes enfants n'aspirent pas à améliorer leur aptitude à apprendre des chansons, pourtant ils progressent assez rapidement. De plus, l'acculturation s'effectue sans enseignement explicite. Par exemple, les adultes n'enseignent pas aux jeunes enfants à mémoriser des chansons, pourtant tous les enfants acquièrent la capacité de mémoriser les chansons de leur culture.

Toujours d'après Chouard, dès qu'on aborde la formation, les préoccupations sont d'un tout autre ordre. Il est alors question d'expériences spécifiques, lesquelles ne sont pas communes à l'ensemble des membres d'une culture, mais réservées plutôt à une sous-culture, une catégorie spécifique de gens pour qui le désir d'accéder à l'excellence, dans une compétence particulière, est encouragé. En construisant sur les bases générales de l'acculturation, l'enfant peut parvenir à développer des habiletés spécialisées, appelées la « compétence de l'expert ». D'une manière générale, on peut dire qu'au sein de notre culture occidentale, l'acculturation constitue le bagage commun acquis par l'ensemble des membres de la société : appréciation de la tonalité, des rythmes occidentaux, de la phrase et des structures musicales. Ces connaissances se manifestent le plus souvent chez le jeune enfant par sa capacité à chanter le répertoire folklorique enfantin, à frapper des mains et à taper du pied au rythme de la musique, sans compter sa faculté d'apprécier la musique de sa culture et d'en éprouver du plaisir. Quant à la formation, elle implique un processus d'apprentissage conscient qui vise à un plus grand accomplissement dans un domaine spécifique. L'enfant est soumis à une méthode afin d'accroître ses compétences. Les méthodes sont fournies par l'éducation et sont généralement appliquées par un enseignant qualifié. De plus, les méthodes pédagogiques sont souvent sélectionnées pour favoriser une habileté particulière. Lorsqu'il s'agit de formation, il importe de développer des compétences de façon efficace.

\section{PARTICULARITÉS DE L'APPRENTISSAGE INSTRUMENTAL}

Alors que l'habileté à chanter s'acquiert pour la majorité des enfants sans effort conscient particulier, la maîtrise du chant classique et d'un instrument de musique nécessite un entraînement à la fois spécial et prolongé, et une grande motivation. Contrairement à des activités plus spontanées, comme le chant folklorique ou l'écoute de la musique, l'apprentissage formel, requis par le 
chant classique et le jeu instrumental du répertoire savant, demande un effort contrôlé et délibéré, et ce type de travail devient vite désagréable pour les enfants, surtout lorsque cet effort doit être fourni régulièrement. Quelque noble que soit l'intention, dans la méthode Suzuki, d'éveiller la motivation du jeune enfant avant les débuts de l'apprentissage instrumental, cette motivation disparaît rapidement lorsque l'élève est confronté à l'effort et au travail ardu exigé pour arriver à la maîtrise technique requise dès le commencement. Ce n'est pas tellement la motivation du jeune enfant qui conduit à la poursuite des études musicales, mais la détermination du parent et, dans bien des cas, une certaine forme de coercition qui oblige à poursuivre les leçons. Laissés à eux-mêmes, bien peu d'enfants parviendraient à s'astreindre à la pratique quotidienne. L'apprentissage formel est freiné par les problèmes de motivation, d'ennui, de résistance de la part de l'enfant, et c'est surtout grâce à l'ingéniosité du parent qui tente de maintenir l'intérêt de l'enfant que celui-ci progresse. Toute personne assistant à une rencontre de parents dans le programme Suzuki sera immédiatement convaincue de cette réalité. Cela ne veut pas dire que le manque de motivation et la coercition sont davantage présents dans la méthode Suzuki, mais que les préoccupations pratiques de la méthode Suzuki, comme de toutes les autres méthodes, sont bien éloignées du processus naturel d'acquisition d'une langue. L'enfant qui apprend à parler ne rencontre aucun problème de motivation et le processus d'acquisition de la langue s'installe même lorsque les parents portent peu attention aux réalisations linguistiques de leur enfant.

Une autre distinction importante doit être faite entre le degré de perfection exigé par les deux habiletés que sont le langage et le jeu instrumental classique. Dans le premier cas, on recherche la capacité de pouvoir communiquer avec les siens; la maîtrise des subtilités et la richesse de la langue demeurent une compétence spécialisée que seul un petit groupe social atteindra. En musique, par contre, un haut niveau de compétence est exigé dès le début des études musicales, tant au plan de la maîtrise technique qu'à celui de la justesse de l'expression. L'assimilation des normes culturelles d'interprétation de la musique savante est un processus très sophistiqué où l'interprétation est constamment raffinée afin de se rapprocher d'un idéal rarement recherché dans la communication verbale courante.

La méthode Suzuki laisse également supposer que la clé du succès repose sur l'écoute de la cassette. Si cette écoute est très importante pour développer le sens musical, elle n'est pas suffisante pour apprendre à jouer la musique savante. Si cet élément importe et contribue à la réussite, il ne l'assure pas à lui seul. Les progrès de l'élève ne sont pas proportionnels au nombre d'heures d'écoute; l'apprentissage d'un instrument ou du chant classique est beaucoup trop complexe pour dépendre d'un facteur unique. La motricité, la coordination, la concentration, l'intelligence sont des éléments de première importance. Il serait trompeur de suggérer, en présence de progrès plutôt lents ou d'obstacles difficiles à surmonter, que l'augmentation des heures d'écoute soit une solution assurée. 


\section{ConClusion}

La valeur d'une première éducation musicale axée sur l'oreille a été maintes fois affirmée depuis plus de trois siècles; aujourd'hui, plusieurs pédagogues défendent les bienfaits d'un apprentissage instrumental basé sur une démarche en trois étapes : écouter, jouer, lire. Si Suzuki n'a pas innové en proposant une approche auditive, il a toutefois le mérite d'avoir permis, au cours des cinquante dernières années, la diffusion de ce genre d'approche partout à travers le monde.

Il ne fait aucun doute que l'importance accordée à l'apprentissage par oreille au début des études musicales est une des grandes richesses de la méthode Suzuki. L'idée que l'enfant soit invité à se familiariser avec les mélodies, les rythmes, l'harmonie, les phrases musicales en se servant de son oreille, et non du code écrit, est intéressante à plusieurs égards. Mais l'apprentissage d'un instrument comprend plus que l'apprentissage du langage musical et repose sur une composante motrice très difficile à acquérir. Alors qu'apprendre à marcher est une habileté motrice qui s'acquiert par un processus naturel, apprendre à manger avec un ustensile ou apprendre à faire de la bicyclette sont des apprentissages beaucoup moins naturels puisqu'ils impliquent la maîtrise technique d'un outil extérieur au corps. La maîtrise du jeu instrumental relève davantage de cette deuxième catégorie.

J'ai voulu par ce texte démontrer les limites épistémologiques de la pensée métaphorique véhiculée par la méthode Suzuki. Cette pensée est riche parce qu'elle permet de mieux comprendre certaines pratiques pédagogiques en faisant des rapprochements entre différentes composantes. Mais, en soulignant les ressemblances entre deux objets, la pensée métaphorique tend à passer trop rapidement sur les différences spécifiques. Ainsi, en faisant l'éloge de l'apprentissage naturel de la langue maternelle, la méthode Suzuki ignore les spécificités de l'apprentissage de la musique savante. Le rapprochement entre l'apprentissage d'un instrument de musique et celui de la langue maternelle est certes séduisant, mais peut aussi être trompeur. Car, si le modèle de l'apprentissage de la langue maternelle est valable pour expliquer le phénomène de l'acculturation musicale, il peut devenir insidieux quand on l'applique à l'apprentissage d'un instrument musical.

Cette recherche n'a porté que sur le premier principe de la méthode Suzuki, le mother-tongue approach. Il serait important de soumettre à l'analyse critique les autres principes de cette méthode et d'en confronter les orientations pédagogiques aux données empiriques de la psychologie de la musique. Nous pourrions ainsi obtenir une vision plus juste des idées véhiculées par cette importante méthode, tout en étant en mesure de confirmer, de nuancer ou d'infirmer les intuitions pédagogiques de Shinichi Suzuki.

\section{Résumé}

Dans la méthode Suzuki, un parallèle est proposé entre l'apprentissage de la langue maternelle et celui d'un instrument de musique. De ce rapprochement se dégage toute une série de principes qui orientent chacun des aspects de la 
méthode. Si le concept de l'apprentissage de la langue maternelle est une métaphore intéressante pour expliquer, en termes simples et imagés, la pédagogie privilégiée, un parallèle direct entre les deux types d'apprentissage suggère une simplicité illusoire de l'apprentissage de l'instrument. Cet article veut baliser les limites de la métaphore.

\begin{abstract}
A parallel is proposed between learning the mother tongue and learning the language of a musical instrument via the Suzuki method. This comparison generates a whole series of principles that orientate each aspect of the method. Although the concept of learning the mother tongue is an interesting metaphor to provide a simple and picturesque explanation of the pedagogy in question, nonetheless, a direct parallel between the two types of learning implies deceptively that learning the instrument is simple. This article aims to delineate the limits of the metaphor.
\end{abstract}

